\title{
Incidence and determinants of mortality among HIV infected adult patients on antiretroviral therapy in Ethiopia: A systematic review and Meta-analysis
}

\section{Habtamu Lijalem}

Wolaita zonal health department

Shimelash Bitew Workie ( $\nabla$ sbitew0@gmail.com )

Wolaita sodo university

Mohammed Suleiman

School of anesthesia, College of Health Sciences and Medicine, Wolaita Sodo University

Muluken Gunta Gutulo

Wolaita zonal health department

\section{Takele Tadesse}

School of Public Health, College of Health Sciences and Medicine, Wolaita Sodo University

\section{Research}

Keywords: Highly Active Antiretroviral therapy, incidence, AIDS related death, survival, adult HIV patients and Ethiopia

Posted Date: May 8th, 2020

DOl: https://doi.org/10.21203/rs.3.rs-26499/v1

License: (c) (i) This work is licensed under a Creative Commons Attribution 4.0 International License. Read Full License 


\section{Abstract \\ Background}

Antiretroviral therapy has been highly associated with reduction in the incidence of mortality in HIV/AIDS patients over time. However, there is a regional variation in the extent of reducing the incidence of mortality in many developing countries including Ethiopia. Hence, this study was conducted to generate summary evidences-based data for incidence of mortality and determinants of mortality.

\section{Methods}

Articles were comprehensively searched on Pub Med, Google Scholar, Cochrane library, Scopus, and DOAJ databases using Boolean operators. A Dersimonian and Laird methods of random effect model was used to estimate incidence and determinants of mortality. Heterogeneity, publication bias and quality of each study were checked. Subgroup analysis was employed. Relevant data from each study were extracted. STATA software version 14 was used for all statistical analysis.

\section{Result}

A total of 21 articles were finally reviewed and analyzed. Incidence of mortality was found to be 5/100person year of observation (95\% Cl: 4-5/100pyo). Most of the death (67\%) occurred during the first year of HAART initiation. Baseline Advanced WHO clinical stage (PHR (Pooled Hazard Rate) 2.88; 95\%Cl: 2.23.8), low CD4 cells count (PHR 1.88; 95\% Cl: 1.5-2.4), low body weight (PHR 1.6; 95\% Cl: 1.2-2.2), low hemoglobin level (PHR 2.4; 95\% Cl: 1.7-3.4), presence of TB infection (PHR 2.9; 95\% Cl: 2.13-4.61), non - working functional status (PHR 3.9; 95\% Cl: 2.8-5.4), bad medication adherence (PHR 4.8; 95\% Cl: 3.27.2), lack of cotrimoxazole preventive therapy (PHR 1.5; 95\% Cl: 1.2-2.0), being male (PHR 1.4; 95\% Cl: 1.2-1.8) and older age (PHR 1.2; 95\% Cl: 1.04-1.41) were significantly associated with increased mortality in this study.

\section{Conclusion}

Incidence of mortality was high particularly early in the course of therapy. Advanced WHO clinical stage, CD4 cells count low body weight, low hemoglobin level presence of TB infection, bad medication adherence older age and non-working functional status were significant determinants of incidence of mortality. Comprehensive service and strict follow up should be given to avert this high rate of mortality.

\section{Background}

Human Immune virus (HIV) infection is spread widely in a short span and became one of the world's most serious health and development challenges in many countries. Seventy-seven million people have 
become infected with HIV and 35 million people died of HIV since the start of the epidemic in 1981. There are 35.1 million [29.6 million-41.7 million] adult populations globally living with HIV, and the total of 1.62 million [1.3 million-2.1 million] adults become newly infected and 830000 [590 000-1.2 million] adults were died in 2017 . Globally $75 \%$ of people living with HIV were aware of their HIV status. The remaining 25\% (over 9 million people) still need access to HIV testing services in 2017. In 2017, 21.7 million people living with HIV (59\%) were accessing antiretroviral therapy (ART) globally, an increase of 2.3 million since 2016 and 8 million in 2010. In 2017, there were 25.7 million people living with HIV $(69 \%)$ in Africa, 5.2 million (14\%) in Asia and the Pacific, and 2.2 million (6\%) in Europe and North America (1 - 4).

Global deaths from Acquired Immune Deficiency Syndrome (AIDS) related illness among people living with HIV has declined from a peak of 1.9 million [1.4-2.7 million] in 2004 to 940000 [670 000-1300 000 in 2017. Since 2010, AIDS-related mortality has declined by $34 \%$ (4). AIDS-related mortality declined by $42 \%$ from 2010 to 2017 in eastern and southern Africa, 24\% in western and central Africa, 39\% in Asia and the Pacific, 36\% western and central Europe and North America, 23\% Caribbean and $12 \%$ in Latin America. Reaching the 2020 milestone will require further declines of nearly 150000 deaths per year (4).

In all region of Africa the total number of people living with HIV infection of all age was 25.92 million, 1.2 million new infections and about 669,800 deaths due to AIDS in 2017. Sub-Saharan Africa regions accounted about $66 \%$ the global burden of the disease (3). In Ethiopia, the total number of people living with HIV in 2018 was 678,165 and new HIV infection in the same year was 20,551 and annual death among adult populations due to HIV/AIDS was 9,294. Among these female's death due to AIDS accounted a total of 5019 (5). In Ethiopia, the incidences of mortality due to AIDS-related causes dropped by 50 percent in the two years following the introduction of free ART in 2005. Between 2001 and 2009, the mortality rate attributable to AIDS fell from $50-14 \%$ for women, and $40-11 \%$ for men $(6,7)$. Every individual will be free of HIV, free from discrimination and with dignity, equality and healthy, and any people infected with HIV should receive the treatment, protection, care and support to survive and improve their quality of life (8).

HIV primarily affects the most productive age group, and it affects not only health of individuals, but also households, communities, and nations. Many of the countries hardest hit by HIV also face serious challenges due to other infectious diseases, food insecurity, and additional global health and development problems. HIV/AIDS has remained one of the top priority's health agenda for the Ethiopian government over last thirty-year period (5).

Clinical benefit of Highly Active Anti-Retroviral Therapy (HAART) for HIV infected patients, in terms of mortality reduction and improved quality of life, is well established but shows regional variations. Higher incidence of mortality within the first years of HAART initiation especially during the first 3-6 months of treatment and determinants contributing to this high mortality are poorly understood. There are several determinants of mortality for HIV infected patients started HAART which include, CD 4 count, total lymphocytes count, body mass index, and medication adherence, WHO clinical stage, years lived with the 
virus, presence of opportunistic infections. Different studies conducted in Ethiopia indicated that there were variations among the determinants of mortality due to AIDS related illness. So, a better knowledge of determinant factors would allow closer follow-up and more targeted interventions for HIV infected patients, thus reduce excess mortality, alter morbidity and improve quality of life (9-19).

There is only one study tried to describe the determinants of mortality by systematic review, but he failed to do meta-analysis (20).Hence, studying the pooled incidence rate and determinants of mortality among HIV infected adult patients who started HAART will provide better evidence-based quality data that are important for the healthcare system of the country, particularly for ART program.

\section{Method}

\section{Protocol and Registration}

This systematic review and meta-analysis were conducted in accordance with the Preferred Reporting Items for Systematic review and Meta-Analysis (PRISMA) statement of 2009. The protocol for this systematic review and meta-analysis was registered in the international prospective register of systematic reviews (PROSPERO) which can be accessed using http://www.crd.york.ac.ku/PROSPERO with the registration number CRD42019123380.

Eligibility criteria

This systematic review and Meta-analysis considered all those published and unpublished studies conducted throughout Ethiopia (Table1).

Inclusion criteria

All studies done by Observational studies conducted using cross-sectional, case-control and both retrospective and prospective cohort study design. Studies conducted in Ethiopia at any health facilities that provide HAART. Studies reported incidence and/or determinants of mortality among adult population above 15 years of age who is infected with HIV. Studies with the outcome of interest are mortality due to AIDS related causes. Published and unpublished studies written in English language from January 1 , 2005 up to April 30, 2019.

\section{Exclusion criteria}

Studies without the outcome of incidence and/or determinants of mortality and Program evaluation studies; Studies conducted on adult HIV infected patients who are on Pre-ART and Studies that will not provide full information/ missed data were excluded.

Information sources

Literature search strategies were searched from free databases Google scholar, Pubmed, Scopus, Cochrane library and Directory of Open Access journal. University repositories used for searching 
unpublished studies includes the Addis Ababa, University of Gonder, Jimma, Haramaya, Bahir Dar and Hawassa Universities. The research center organizations that were searched for studies on their repositories include African Digital Research Repositories, Economic Commission for Africa, African Union and National Academic Digital Repository of Ethiopia. The search terms used for institutions was directly inserting the title into the search engine in each institution. "Incidence and determinants of mortality among HIV infected adult patients who initiated HAART in Ethiopia". The search was performed from March 15 - April 30, 2019.

\section{Literature Searching strategy}

A comprehensive internet-based search was conducted to find research articles on the electronic databases with keywords and MESH terms stringed out using "AND", "OR" and "NOT" Boolean operators. Search was used based on COCOPO (Table1). The findings from the electronic freely access databases, hand search of the reference lists and institutional repositories were pooled for subsequent selection. We have searched articles from the reference list of the included studies directly from the internet.

Study selection

All retrieved studies were exported to Endnote X8 reference manager (Thomson Reuters) and duplicate studies were removed. The remaining studies were screened based on their titles and abstracts and then a full-text review was performed to determine their eligibility. Lastly, studies that fulfilled the eligibility criteria were included in the final review and synthesis.

\section{Data collection process}

Quantitative data were extracted from papers included in the review using the standardized data extraction tool. Data was collected from April 30,2019 to March 30,2019. Two reviewers MPH in Epidemiology have extracted the data and cross-checked to ensure consistency from all included studies. The following Information's author and year of publication, study area, study subjects, study design, the total sample size, the number of participants and events in each groups, median follow-up period, incidence of mortality due to AIDS related causes, and Hazard Ratio among exposed and non-exposed groups were extracted. There was no discrepancy happening during data extraction between the reviewers. Standardized data extraction tool from Joanna Briggs Institute Meta-Analysis of Statistics Assessment and Review Instruments were used

Data quality control measures

Quality of all studies included in the review and analysis was assessed and evaluated using Joanna Briggs Institute (JBI 2017) quality appraisal tools adapted for studies reporting observational studies. Based on the quality assessment tools all the included studies scored above $82 \%$. Search beyond published literature was done to reduce the risk of publication bias.

Synthesis of results 
A DerSimonian and Laird method of random-effect model was employed to compute the overall pooled incidence of mortality with its corresponding $95 \%$ confidence interval. The pooled hazard ratios of the determinants of mortality were estimated from each study. Heterogeneity across studies was assessed using the Cochrane $Q$ test and quantified by Higgins and Thompson's $I^{2}$ statistic. Subgroup and sensitivity analysis were performed to explore the possible causes of heterogeneity. Publication bias across studies was checked using funnel plot and Egger's regression test. STATA software version 14 was used for all statistical analysis. The results of the analysis were summarized and presented graphically using Forest Plot.

Operational definitions

Incidence of mortality: Incidence rate was defined as the rate of number of participants died during the follow up period to the total person year of observation. (events/person years of observation).

WHO clinical stage: Study participants are classified into two groups based on WHO categories. Those patients with WHO clinical stage 1 and 2 are categorized as Non-exposed group and those patients who satisfied WHO clinical stage 3 and 4 are as exposed group.

CD4+ cells count: Patients with baseline CD 4 cells count less than 200 cells/ $\mu \mathrm{l}$ was considered as exposed group while those patients with CD 4 cells count greater than 200 cells/ $\mu$ l was considered as unexposed group.

Adherence: Patients who had good medication adherence was classified as non-exposed group and those patients with poor and fair adherence were classified as exposed group.

Functional status: Patients who were classified as working functional status at enrollment to chronic care are grouped into non-exposed group and those ambulatory and bedridden patients are exposed group.

Anemia: Patients with hemoglobin level below $10 \mathrm{~g} / \mathrm{dl}$ had anemia and classified as exposed grouped whereas patients with hemoglobin level above $10 \mathrm{~g} / \mathrm{dl}$ had no anemia and classified as non-exposed grouped.

Body weight: patients who had baseline body weight lower than $45 \mathrm{~kg}$ were assigned to exposed group and above $45 \mathrm{~kg}$ as non-exposed group.

Age: patients whose age was above 40 years were categorized as exposed group and below 40 years as non - exposed group.

\section{Results}

\section{Description of studies}


A total of 423 articles were retrieved. After removing duplicated retrievals, 423 articles remained, of which 392 were removed during initial assessment due to exclusion criteria's set at the review. From the remaining 31 articles 10 were removed based full text articles. Finally, 21 articles were included for the final synthesis (figure 1). All articles included in the final reviews and analyses were retrospective cohort studies which were conducted throughout the country from September 01, 2006 up to October 25, 2018.

\section{Study characteristics}

The studies comprised a total sample size of 23904 participants ranging from 152 (ID 08) to 5664 (ID 11) that contributed to a total of 63, 107.7 person years of observations during their follow up. Of these, 13390 (56\% of the participants) were females. There were 2, 804 events occurred in the study period. The study contributed a total of 1154.75 months of follow up period, a median follows up period of 60 months ranged from a minimum of 55 weeks to a maximum of 96 months.

\section{Incidence of mortality}

Incidence of mortality analyzed from eighteen studies. The random pooled estimate of the incidence of mortality among adult HIV infected patients after HAART initiation was 5 deaths per 100 person years of observation (95\% CI: 4 - 5/100pyo, p-value < 0.001; heterogeneity $\mathrm{I}^{2}=97.39 \%$ ) (figure 2 ).

\section{Subgroup analysis of incidence of mortality}

It was subgrouped based on publication year. Incidence of mortality among HIV infected patients from 2005 - 2010 was 8 per 100 person years of observation (95\% CI: 6 $11 / 100$ pyo). There was no difference in the pooled estimate of incidence of mortality among HIV infected adult patients between the years $2011-2014$ (incidence rate $=4$ per100pyo; 95\% CI: 3 - 6/100pyo, p-value < 0.01; heterogeneity $\mathrm{I}^{2}=98.49 \%$ ) and $2015-2019$ (incidence rate $=4$ per 100pyo; 95\% CI: $3-5 / 100$ pyo, p-value $<0.01$; heterogeneity $\mathrm{I}^{2}$ $=95.43 \%$ ), (figure 3). There was still considerable level of heterogeneity even after subgroup analysis-based publication year, number of participants, person year observation. Hence moderators could not be a source of heterogeneity.

\section{Sensitivity analysis of incidence of mortality}

The leave - one - out sensitivity analysis has been performed for incidence of mortality. The sensitivity analysis confirmed that the finding of this study was independent of a single study. The pooled estimated incidence of mortality varied between 4.0 deaths per 100 person year of observation (95\% CI: 3.0 - 5.0) and 5.0 deaths per 100 person year of 
observation (95\% CI: 4.0 - 6.0) after removing one study at a time from the analysis (table 2).

\section{Determinants associated with incidence of mortality among adult on HAART}

\section{WHO Clinical Stage of the Patients}

Advanced WHO clinical stage (stage III and IV) were 2.9 times more likely to die early than WHO clinical stage I and II. The pooled hazard ratio of patients with WHO clinical stage III \& IV was 2.88 (95\% CI: 2.20 - 3.7), $\mathrm{p}$ - value < 0.001; heterogeneity $\mathrm{I}^{2}=81.4 \%$ ). A subgroup analysis indicated that the hazard ratio of mortality among HIV infected adult patients presented with WHO clinical stage III \& IV before and after the year 2014 was 2.93 (95\% CI: 1.98 - 4.35, p - value < 0.001; heterogeneity $\left.\mathrm{I}^{2}=77.7 \%\right)$ and 2.84 (95\% CI: 1.94 $4.15, \mathrm{p}$ - value $<0.001$; heterogeneity $\mathrm{I}^{2}=84.5 \%$ ), respectively sensitivity analysis was done but no change on Hazard ratio (figure 4).

\section{Baseline CD4 Cells count}

Baseline CD4 cells count had significant association with Mortality. The pooled hazard ratio of patients with low CD4 cell count at the initiation of HAART was 1.88 (95\% CI: 1.50 - 2.36, p - value < 0.001; heterogeneity $\mathrm{I}^{2}=77.5 \%$ ). A subgroup analysis indicated higher hazard of dying before 2015 than after 2015. The hazard of dying among patients with low CD4 count before and after the year 2015 was 2.01 (95\% CI: $1.53-2.65$, p - value < 0.001; heterogeneity $\left.\mathrm{I}^{2}=79 \%\right)$ and 1.69 (95\% CI: $1.11-2.57, \mathrm{p}$ - value $=0.002$; heterogeneity $\mathrm{I}^{2}$ $=74 \%$ ), respectively (figure 5) sensitivity analysis was done but no change on Hazard ratio.

\section{Functional Status}

Bedridden functional status had 3.9 more likely to death than others at baseline. Overall pooled hazard ratio of patients presented with bedridden functional status was 3.90 higher than patients presented to care in working functional status (95\% CI: $2.81-5.42$, p value of $<0.001$; heterogeneity $\mathrm{I}^{2}=92 \%$ ) (Figure 6). Sensitivity analysis was done but no change on Hazard ratio.

\section{ART adherence}

The pooled hazard ratio of patients with bad ART adherence was 4.81 (95\% CI: 3.19 - 7.26, p-value of $<0.001$; heterogeneity $\mathrm{I}^{2}=95.2 \%$ ). The pooled hazard ratio among patients with improper medication adherence before and after the year 2015 was 4.95 (3.05 - 8.01, p - 
value < 0.001; heterogeneity $\left.\mathrm{I}^{2}=96.2 \%\right)$ and $4.51(1.61-12.60, \mathrm{p}-$ value < 0.001; heterogeneity $\mathrm{I}^{2}=94 \%$ ), respectively (figure 7 ). Sensitivity analysis was done but no change on Hazard ratio.

\section{Tuberculosis co-infection}

Tuberculosis co-infected patients had 2.9 times to die than non-infected (2.92 (95\% CI: 2.31 - 4.01)). Heterogeneity test indicated $I^{2}=91.7 \%$, hence random effect was assumed during analysis (figure 8). Sensitivity analysis was done but no change on Hazard ratio.

\section{Anemia}

Overall pooled hazard ratio of patients with low hemoglobin level at initiation of HAART was 2.42 (95\% CI: 1.72 - 3.39, p-value < 0.001; heterogeneity $\mathrm{I}^{2}=89.8 \%$ ). The pooled hazard ratio of patients with low hemoglobin level before and after the year 2015 was 2.46 (95\% CI: 1.84 - 3.29, p-value < 0.001; heterogeneity $\mathrm{I}^{2}=76.7 \%$ ) and 2.34 (95\% CI: 0.91 $6.05, \mathrm{p}$-value $<0.001$; heterogeneity $\mathrm{I}^{2}=96.1 \%$ ), respectively. In this subgroup analysis, baseline hemoglobin level was not a significant determinant of mortality after the year 2015 (figure 9). Sensitivity analysis was done but no change on Hazard ratio.

\section{Cotrimoxazole Preventive Therapy (CPT)}

The overall pooled hazard ratio of patients who did not take CPT was 1.53 (95\% CI: 1.16 2.02, p-value < 0.001; heterogeneity $\mathrm{I}^{2}=79.3 \%$ ). The pooled estimate of hazard ratio in the year before and after 2015 was 1.70 (95\% CI: 1.14 - 2.52, p - value < 0.001; heterogeneity $\left.\mathrm{I}^{2}=86 \%\right)$ and 1.29 (95\% CI: $0.95-1.75, \mathrm{p}-$ value $=0.245$; heterogeneity $\mathrm{I}^{2}=29 \%$ ), respectively (figure 10). Sensitivity analysis was done but no change on Hazard ratio.

\section{Low body weight}

The overall pooled hazard ratio of patients with low body weight at the initiation of antiretroviral treatment was 1.59 higher than those patients with normal body weight (95\% CI: 1.15 - 2.19, p -value < 0.001; heterogeneity $\mathrm{I}^{2}=88.6 \%$ ), (figure 11 ). Sensitivity analysis was done but no change on Hazard ratio.

\section{Gender}

Being male gender had $41 \%$ more risk of dying than being female gender $(1.41$ (95\% CI: 1.19 - 2.67, $\mathrm{p}$-value $<0.001$; heterogeneity $\left.\mathrm{I}^{2}=81.1 \%\right)$ ( figure 12 ). Sensitivity analysis was done but no change on Hazard ratio. 
The overall pooled hazard ratio of patients above 40 years was 1.21 (95\% CI: $1.04-1.41, \mathrm{p}$ value $=0.031$; heterogeneity $\mathrm{I}^{2}=48.2$ ) (figure 13 ). Sensitivity analysis was done but no change on Hazard ratio.

\section{Publication Bias}

The studies were scattered symmetrically in funnel plot which indicated that there was no publication bias among the studies (figure 14).

\section{Egger Test}

The estimated publication bias coefficient of egger test was 0.5 with a standard error of 1.36 and p-value of 0.716 which indicated that there is no evidence for the presence of small study effect among the included studies (Table 3).

\section{Discussion}

In this review, the summary incidence of mortality among adult HIV infected patients after taking HAART in Ethiopia was 5 deaths per 100 person years of observation (95\% Cl: $4-5 / 100$ pyo). The pooled incidence of mortality has decreased from 8 deaths per 100 pyo in 2010 to 4 deaths per 100 pyo in 2018 . This indicated that there is an improvement in reduction of mortality among HIV infected patients through times. This might be due to improvement in health care delivery systems; commitments from the government and stakeholders to achieve the global target; improved quality of life of the patients due to treatment, care and support.

Pooled incidence of mortality in this review was low compare to Central African Republic (9.1/100pyo, South Sudan 8.2/100pyo, Côte d'Ivoire 7.5/100pyo, Cameroon 7.2/100pyo, Chad 7.1/100pyo and Nigeria $6.5 / 100$ pyo done via systematic review and meta-analysis (34). The variation may be due to difference in study setting, difference in their culture, economic status, and difference in the level of awareness and educational background of the patients between these populations.

However, this result is high compared to study done on 200000 patients in Botswana National Program from 2002-2012 indicated that lower incidence of mortality rate which was 2.06 deaths per 100 person years of observation over a total follow up period (35). This difference might be difference in the follow up period, early detection and diagnosis of the infection and then early rapid scale up of ART before CD4 deterioration and development of advanced WHO clinical stage starting from 2002 in Botswana may explain the attributable difference in the estimation.

This review demonstrated trends of reduced death rates from 8 per 100 pyo in the year group 2005 2010 to 4 per 100 pyo in the year group $2015-2018$. This result is similar with the trend analysis of mortality among ART treated HIV infected adult in Asia-pacific region between 1999 to 2017 indicated 
that the overall incidence rate of mortality during follow up period was 0.28 per 100 pyo. Moreover, the incidence rates of AIDS - related deaths have been decreased from 0.51/100 PYS in the year groups 2003 - 2007 to 0.09/100 PYS in the year group $2013-2017$ ( $p<0.001)$ in Asia - pacific region (35) and from $7-2 \%$ in Botswana in the year $2002-2012$ (34,35). The improvements in the reduction of mortality through time have been related with the introduction of effective free HAART to all HIV infected patients before the deterioration of CD4 cell count and development of advanced AIDS stages, which extends the time to AIDS deaths and decrease the total number of AIDS death itself

Patients presented with advanced WHO clinical stage, low CD4 cells count, low body weight, low hemoglobin level, presence of TB co-infection, non - working functional status, improper medication adherence, lack of cotrimoxazole preventive therapy, male sex and older age at the initiation of HAART were significantly associated with increased mortality.

The hazards of dying among HIV infected adult patients presented with WHO clinical stage III and IV at the initiation of HAART were almost three times more likely than those patients with WHO clinical stage I and II. This finding is the same as studies done in LMIC (low and Middle income countries) and Tanzania with OR of $2.3(95 \% \mathrm{Cl}, 1.0-5.5)$ that the vast majority of the patients who were died had advanced HIV disease stage $(36,37)$. People presented with advanced HIV disease are at high risk of opportunistic infections and increased incidence of death, even after starting HAART. Severe immune suppression has frequently shown to be associated with increased risk of fatal advanced HIV disease. Early initiation of treatment before the development of advanced WHO clinical stage of the disease will reduce morbidity and prevent the high mortality rate occurred among patients with advanced WHO clinical stage of the disease $(36,37,38)$.

Patients who had low CD 4 cells counts at initiation of HAART was almost twice more likely to experience hazard of death than patients with higher CD4. This is a similar finding to the systematic review and meta-analysis conducted by Anglemyer et al (39), study at Iran (40), Botswana (35), Tanzania (37) and In trend mortality analysis in Asia - pacific region (36). Immediate HAART Initiation to all HIV infected patients at higher CD4 cells counts has been associated with several health benefits. These may include reduce the risk of mortality, slow progression of the infection to AIDS stage, can improve the likelihood of immunological recovery (CD4 T - Cells counts reaching 800 cells $/ \mathrm{mm}^{3}$ or more after HAART), can increase the percentage of individuals who achieved rapid viral suppression, prolongs survival of the patients and quality of life $(36,37,41)$.

Patients presented with non - working functional status at the initiation of HAART in Ethiopia were four times more likely to experience death than those patients with working functional status. A study done in Maryland, USA to examine the association between physical performance and mortality on HIV infected patients indicated that reduced physical performance among HIV infected individuals had been associated with increased mortality (hazard ratio $2.52,95 \% \mathrm{Cl}: 1.59-4.00)$ (42). HIV infected individuals frequently exposed to physical performance impairment because of loss of muscle mass, altered body composition, decrease physical function, frailty, and disability. These predisposing causes may restrict 
the day to day activities of the patients especial during advanced HIV diseases and increased the likelihood of mortality (39).

Different studies reported that patients with non - adherence to treatment had been associated with increased risk of mortality than those who adhere to their treatment. HAART will work best for patients who adhere to their medication and these patients have increased chance of survival. Poor adherence is associated with the risk of early treatment failure and rapid development of drug resistance $(43,44)$. The hazard of death among adult HIV infected patients with poor medication adherence at initial enrollment to chronic care had nearly five times higher than those patients with good medication adherence.

In this analysis, patients diagnosed with TB at initiation of HAART were three times more likely to die than those patients without TB. This finding is in line with study done in assessing determinants of mortality in South Africa (45), Iran (40) and a meta-analysis study at LMIC (36). This might be due to HIV infected patients co-infected with TB are prone to develop advanced AIDS stage easily as their immune system have been deteriorated. TB and HIV have vicious cycle. Innovative approaches to reduce TB infection should be implemented and scaling up of TB prevention strategy including routine INH provision to all HIV infected adults, rapid TB detection and diagnosis methods and immediate treatment should be strengthened $(36,37)$.

In this review, HIV infected patients with severe anemia at initiation of HAART had two times risk of dying than those patients without anemia. This finding is similar with the finding from Tanzania showed that severe anemia had been associated with increased mortality with odds ratio of $6.6(95 \% \mathrm{Cl}, 3.4-12.9)$ (37). Another study at LMIC indicated that anemic patients with hemoglobin level $<8 \mathrm{gm} / \mathrm{dl}$ have experienced the greatest risk of mortality. Anemia among HIV infected individuals are easily exposed to chronic diseases like chronic heart failure that may be fatal to the patients could be the best explanation for the finding $(35,36)$.

HIV infected patients who did not take a cotrimoxazole preventive therapy before or at the initiation of HAART were $53 \%$ more risk to die than patients who took cotrimoxazole preventive therapy. The finding is similar with systematic review and meta-analysis conducted at LMIC, patients who had taken CPT in their course of therapy had a 58\% reduced incidence of mortality among HIV infected adults (46). This might be CPT has been recommended for all HIV infected patients developing advanced AIDS stage III \& IV and patients with WHO stage I \& II whose CD 4 counts $<350$ cells $/ \mu$ l. Cotrimoxazole is safe, well tolerated, widely available and inexpensive which is used to prevent opportunistic infections which can be developed when the immune system of the body is compromised due to HIV infection (40).

Patients with low body weight at the initiation of HAART had $59 \%$ higher risk of death than those patients with normal range of body weight. A systematic review and meta-analysis conducted in LMIC among adult patients initiating HAART showed that low Body Mass Index/ body weight was independently associated with early mortality (36). In the trend mortality at Asia - Pacific region, lower BMI was a significant determinant associated with increased mortality in which underweight patients with BMI value $<18.5$ had three times more hazard of death than patients who were in the normal BMI range of $18.5-$ 
$24.9(\mathrm{HR}=3.33,95 \% \mathrm{Cl}: 2.31-4.81)(36)$. This may be due to the underlying effects of poor nutrition which may result in low body weight and hence associated with early mortality. Loss of appetite, inability to eat/ difficulty to swallow foods and stress associated with HIV/AIDS make the immune system to weaken and easily vulnerable to advanced AIDS stage. Body weight was used as a proxy indicator of nutritional status of a person.

In this analysis, being male had $41 \%$ higher risk of death due to HIV/AIDS related cause than female after the initiation of HAART in Ethiopia. This finding is consistent with a national ART study conducted at Botswana that predicted women had a lower risk of mortality (about $46 \%$ of reduction) due to AIDS related causes when compared with men (odds ratio $=0.64 ; 95 \% \mathrm{Cl} 0.60-0.69)(35)$. In Tanzania one study indicated that being male had almost two times higher odds of death than females (odds ratio = $1.8,95 \% \mathrm{Cl}: 1.1-3.0)(37)$ and a systematic review and meta-analysis in LMIC revealed that male sex was an independent risk factor associated with early mortality in about $30(60 \%)$ of the included studies (35). The reasons why males were in general more likely to die early in the course of therapy than females may be due to the difference in health seeking behavior, biological differences in ART response resulting in poorer adherence in males, difference in HIV treatment outcomes.

In this study older ages above 40 years had $21 \%$ higher hazard of dying from HIV/AIDS when compared to patients below the age of 40 years. This result is in line with a study done at LMIC, Botswana and China indicated that older age above 40 years had been strongly associated with increased odds of AIDS related mortality $(34,35,47)$. This is probably due to the fact that older people would experience to have poor adherence to ARV drugs because of low level of education and their desire to reduce the burden and stigma on their family associated with HIV/AIDS, older people may be underserved by public health care facility and has been associated with late presentation, diagnostic delays, poorer CD4 immune reconstitution may also explain the finding as is was mentioned in these studies.

\section{LIMITATION OF THE STUDY}

Patients who died at home without registered were considered as alive or loss to follow up which result in underestimation of incidence of mortality. Similarity, patients who died at health facility and recorded in the ART registry were considered as death due to HIV related causes as the exact cause of death was not differentiated routinely and this may overestimate the incidence of mortality.

This review and analysis may not include all the eligible studies because of lack of access to the databases. Only free databases (Pubmed, Google Scholar, Scopus, Cochrane and DOAJ) have been accessed for searching studies.

\section{Conclusions And Recommendations}

In summary, there was high incidence of mortality in this review and meta-analysis early in the course of therapy. Most of the death (67\%) occurred during the first year of follow up after initiation of HAART. Advanced WHO clinical stage III\&IV, low CD 4 cells count below $200 \mu \mathrm{l} / \mathrm{ml}$, low body weight below $40 \mathrm{~kg}$, 
low hemoglobin level, presence of TB infection, non - working functional status, bad medication adherence, lack of cotrimoxazole preventive therapy, male sex and older age at the initiation of HAART were the significant determinants found to be associated with increased mortality in this review and meta analysis.

Routine intensified HIV testing and counseling services and early diagnosis of HIV infected patients and then early initiation of HAART before the deterioration of the functional status of the patients, before the reduction of CD4 cells counts and hemoglobin levels and before the development of advanced WHO clinical stage with continue monitoring of medication uptake and health education on adherence to ART regimens in these patients by all health facilities providing ART service will maximize the effectiveness of HAART and help to save their lives.

Approaches to reduce TB infection should be implemented and scaling up of TB prevention strategy including routine INH provision to all HIV infected adults, rapid TB detection and immediate treatment should be strengthened by health care facility.

Increase the utilization of cotrimoxazole preventive therapy to all eligible patients with adequate health education on its important in preventing opportunistic infections.

Easily accessible and available ART services for male and elderly people should be emphasized by service providers.

Multisectoral response office and stakeholders working on HIV prevention and control program should give emphasis on ART services updates and refreshing health care workers and supportive staffs routinely. This is important to provide comprehensive health package to HIV infected patients at all level of service provision.

\section{List Of Abbreviations}

HIV/AIDS - Human Immunodeficiency Virus/Acquired Immunodeficiency Syndrome

BMI - Body Mass Index

CD4 - Clusters of Differentiation 4

CPT - Cotrimoxazole Preventive Therapy

DOAJ - Directory of Open Access Journal

FDC - Fixed Drug Combination

HAART - Highly Active Antiretroviral Therapy

$\mathrm{JBI}$ - Joanna Briggs Institute 
LMIC- Low and Middle Income Countries

PYO - Person Year of Observation

PRISMA - Preferred Reporting Items for Systematic Review and Meta Analysis

PROSPERO - International Prospective Registration of systematic review

SSA - Sub Saharan Africa

TB - Tuberculosis

TLC - Total Lymphocyte Count

WHO - World Health Organization

\section{Declarations}

Ethical approval and consent to participate: Ethical clearance was obtained from the Wolaita Sodo University, college of health sciences and Medicine Institutional Research Ethics Review Committee.

Consent for Publication: not applicable.

Availability of Data and Material: Not applicable

Financial Disclosure: Authors doesn't receive any funding for this research

Competing interest: the authors declared that they have no conflict of interest.

Author's contribution: HL: Initiated the research, wrote the research proposal, conducted the search, did data entry and analysis and wrote the manuscript. MS: Initiated the research, wrote the research proposal, conducted the research, did data entry and analysis and wrote the manuscript. SB: Initiated the research, wrote the research proposal, conducted the research, did data entry and analysis and wrote the manuscript. TT: Contributed in the write up of proposal, analysis and write up of the manuscript. All are equally contributed.

Acknowledgement: We would like to acknowledge Wolaita Sodo University for providing materials used for the work.

\section{References}

1. Ashwini Shete, Current Trends in HIV/AIDS: J HIV AIDS Infectious Diseases: National AIDS Research Institute, Pune, India, December 05, 2014; vol 1: 1-2. DOI: 10.17303/jaid.2013.102.

2. Global AIDS Update Miles to go-closing gaps, breaking barriers, righting injustices [internet], Geneva, Switzerland, UNAIDS: July 2018. Available from AIDS info website http://aidsinfo.unaids.org/. 
3. Joint United Nations Programme on HIV/AIDS; UNAIDS World AIDS Data 2018. Geneva, Switzerland; 2018 Available from unaids.org.

4. Henry J Kaiser Family Foundation; The global HIV/AIDS epidemic Fact sheet 2018. San Francisco, California, KFF; July 2018.

5. Yibeltal Assefa, HIV/AIDS in Ethiopia: an Epidemiologic synthesis: $2^{\text {nd }}$ edition, Federal HIV/AIDS Prevention and Control Office Addis Ababa, Ethiopia; 06 November 2015. Vol 1: 32-36. DOI:10.13140/RG.2.1.1891.5283.

6. Ethiopian public health institute; HIV Related Estimates and Projections for Ethiopia-2017. March 2017. Addis Ababa, Ethiopia.

7. Ethiopia Demographic and Health Survey 2012. Addis Ababa, Ethiopia and Calverton, Maryland, USA, Central Statistical Agency [Ethiopia] and ICF International, 2011

8. Joint United Nation Programme on HIV/AIDS; UNAIDS Strategy for 2016-2021: On the fast-track to end HIVAIDS; Geneva, Switzerland. August 03, 2015.

9. Assefa et al. Outcomes of antiretroviral treatment program in Ethiopia: Retention of patients in care is a major challenge and varies across health facilities; A retrospective longitudinal study. BMC Health Services Research 2011, 11(81):3-5 doi: 10.1186/1472-6963-11-81.

10. Shibre Mengesha et al. (2014) Predictors of Survival in HIV-Infected Patient after Initiation of HAART in Zewditu Memorial Hospital, Addis Ababa, Ethiopia: retrospective cohort study. Hindawi Publishing Corporation International Scholarly Research Notices Volume 2014, Article ID 250913: dx.doi.org/10.1155/2014/250913.

11. Tadesse K, Haile F, Hiruy N (2014) Predictors of Mortality among Patients Enrolled on Antiretroviral Therapy in Aksum Hospital, Northern Ethiopia: A Retrospective Cohort Study. PLoS ONE 9(1): e87392. doi:10.1371/journal.pone.0087392.

12. Mamo Wubshet et al. (2012) High Loss to follow up and Early Mortality Create Substantial Reduction in Patient Retention at Antiretroviral Treatment Program in North-West Ethiopia. Retrospective cohort. International Scholarly Research Network ISRN AIDS Volume 2012, Article ID 721720, doi:10.5402/2012/721720;

13. Biadgilign et al. (2012) Predictors of mortality among HIV infected patients taking antiretroviral treatment in Ethiopia. Retrospective cohort study. AIDS Research and Therapy 2012, 9:15: doi: 10.1186/1742-6405-9-15.

14. Damtew et al. (2014) Survival and Determinants of Mortality in Adult HIV/AIDS Patients Initiating Antiretroviral Therapy in Somali Region, Eastern Ethiopia: cohort study. J AIDS Clin Res 2014, 5:7: DOI: 10.4172/2155-6113.1000327

15. Farahani et al. Trends and determinants of survival for over 200000 patients on antiretroviral treatment in the Botswana National Programme: 2002 - 2013, J Acquir Immune Defic Syndr 2016; 30:477 - 485, DOI:10.1097/QAD.0000000000000921

16. Abebe TW, Chaka TE, Misgana GM, Adlo AM (2016) Determinants of Survival among Adults on Antiretroviral Therapy in Adama Hospital Medical College, Oromia Regional state, Ethiopia. J HIV 
AIDS 2(1): doi http://dx.doi.org/10.16966/2380-5536.117.

17. Tadege (2018) Time to death predictors of HIV/AIDS infected patients on antiretroviral therapy in Ethiopia. BMC Res Notes (2018) 11:761: org/10.1186/s13104-018-3863-y.

18. Tachbele E and Ameni G (2016) Survival and predictors of mortality among human immunodeficiency virus patients on anti-retroviral treatment at Jinka Hospital, South Omo, Ethiopia: a retrospective cohort study. Epidemiology and Health; Volume: 38, Article ID: e2016049: org/10.4178/epih.e2016049.

19. Jerene et al. (2006) Predictors of early death in a cohort of Ethiopian patients treated with HAAR. BMC Infectious Diseases 2006, 6:136: doi: 10.1186/1471-2334-6-136.

20. Biset Ayalew M. Mortality and its predictors among HIV infected patients taking antiretroviral treatment in Ethiopia: a systematic review. AIDS research and treatment. 2017;2017.

21. Mitiku Teshome Hambisa et al. (2013) Determinants of Mortality among HIV Positives after Initiating Antiretroviral Therapy in Western Ethiopia: A Hospital-Based Retrospective Cohort Study, Volume 2013, Article ID 491601, 7 pages, http://dx.doi.org/10.1155/2013/491601. Hindawi Publishing Corporation, ISRN AIDS

22. Andinet Worku Alemu \& Miguel San Sebastián (2010) Determinants of survival in adult HIV patients on antiretroviral therapy in Oromiyaa, Ethiopia, Global Health Action, 3:1, 5398, DOI:

10.3402/gha.v3i0.5398

23. Tesfaye Setegn et al. (2015) Predictors of Mortality among Adult Antiretroviral Therapy Users in Southeastern Ethiopia: Retrospective Cohort Study: Hindawi Publishing Corporation; AIDS Research and Treatment; Volume 2015, Article ID 148769, 8 pages; http://dx.doi.org/10.1155/2015/148769

24. Dinberu Seyoum et al. (2017) Risk Factors for Mortality among Adult HIV/AIDS Patients Following Antiretroviral Therapy in Southwestern Ethiopia: An Assessment through Survival Models; J. Environ. Res. Public Health 2017, 14, 296; doi:10.3390/ijerph14030296

25. Hailemariam S, Tenkolu G, Tadese H, Vata PK (2016) Determinants of Survival in HIV Patients: A Retrospective Study of Dilla University Hospital HIV Cohort. Int J Virol AIDS 3:023

26. Wondimu Ayele et al. (2015) Treatment outcomes and their determinants in HIV patients on Antiretroviral Treatment Program in selected health facilities of Kembata and Hadiya zones, Southern Nations, Nationalities and Peoples Region, Ethiopia: BMC Public Health (2015) 15:826; DOI 10.1186/s12889-015-2176-5

27. Eyuel Tsegaye, Alemayehu Worku (2011) Assessment of antiretroviral treatment outcome in public hospitals, South Nations Nationalities and Peoples Region, Ethiopia: J. Health Dev. 2011;25(2)

28. Tadele A, Shumey A, Hiruy N (2014) Survival and Predictors of Mortality among Adult Patients on Highly Active Antiretroviral Therapy at Debre-Markos Referral Hospital, North West Ethiopia; A Retrospective Cohort Study. J AIDS Clin Res 5: 280. doi:10.4172/2155-6113.1000280

29. Bezalem Eshetu Yirdaw, Eshetu Wenchek (2014) Survival longevity of adult AIDS patients under ART: A case study at Felege-Hiwot Referral Hospital, Bahir-Dar, Ethiopia; J. Health Dev. 2014;28(2):105-115 
30. Mebrahtu Abay et al. (2017) Effect of late HIV diagnosis on HIV-related mortality among adults in general hospitals of Central Zone Tigray, northern Ethiopia: a retrospective cohort study; HIV/AIDS Research and Palliative Care 2017:9 187-192; http://dx.doi.org/10.2147/HIV.SI41895

31. Melkam Amsalu (2015) Survival Status among People Living with HIV/AIDS on Highly Active Anti Retroviral Therapy at Federal Police Referral Hospital, Ethiopia, Retrospective Cohort study: Addis Ababa university; June, 2015

32. Tegiste Assefa, Eshetu Wenchek (2012) Survival analysis of patients under chronic HIV-care and antiretroviral treatment at Tikur Anbessa Specialized Hospital, Addis Ababa, Ethiopia: J. Health Dev. 2012;26(1):22-29

33. Abdo Bedru (2009) Assessment of predictors of survival in patients living with HIV/AIDS after the advent of highly active antiretroviral therapy in Addis Ababa Ethiopia; June 2009

34. Granich R, Gupta S, Hersh B, Williams B, Montaner J, Young B, et al. (2015) Trends in AIDS Deaths, New Infections and ART Coverage in the Top 30 Countries with the Highest AIDS Mortality Burden; 1990-2013. PLoS ONE 10(7): e0131353. doi:10.1371/journal.pone.0131353

35. Gupta A, Nadkarni G, Yang W-T, Chandrasekhar A, Gupte N, et al. (2011) Early Mortality in Adults Initiating Antiretroviral Therapy (ART) in Low- and Middle-Income Countries (LMIC): A Systematic Review and Meta-Analysis. PLoS ONE 6(12): e28691. doi:10.1371/journal.pone.0028691.

36. Jung IY et al. Trends in mortality among ART-treated HIV-infected adults in the Asia-Pacific region between 1999 and 2017: results from the TREAT Asia HIV Observational Database (TAHOD) and Australian HIV Observational Database (AHOD) of leDEA Asia-Pacific. Journal of the International AIDS Society 2019, 22:e25219. doi.org/10.1002/jia2.25219.

37. Daniel Wilfred Gunda et al. Prevalence and Risk Factors of Mortality among Adult HIV Patients Initiating ART in Rural Setting of HIV Care and Treatment Services in North Western Tanzania: A Retrospective Cohort Study. Journal of Sexually Transmitted Diseases 2017, doi.org/10.1155/2017/7075601.

38. Ford et al. Managing Advanced HIV Disease in a Public Health Approach. Clinical Infectious Diseases 2018; 66(S2):S106-10, DOI: 10.1093/cid/cix1139.

39. Anglemyer et al. Early initiation of antiretroviral therapy in HIV-infected adults and adolescents: a systematic review, J Acquir Immune Defic Syndr AIDS 2014, 28 (Suppl 2):S105-S118, DOI:10.1097/QAD.0000000000000232.

40. Mirzaei M, Farhadian M, Poorolajal J, Kazerooni PA, Tayeri K, Mohammadi Y. Survival rate and the determinants of progression from HIV to AIDS and from AIDS to the death in Iran: 1987 to 2016. Asian Pac J Trop Med 2019; 12(2): 72-78.

41. Song A, Liu X, Huang X, Meyers K, Oh D-Y, Hou J, Xia W, Su B, Wang N, Lu X, Xia H, Yang X, Chen H and Wu H (2018) From CD4-Based Initiation to Treating All HIV-Infected Adults Immediately: An Evidence- Based Meta-analysis. Front. Immunol. 9:212. doi: 10.3389/fimmu.2018.00212.

42. Greene et al. The relationship of physical performance with HIV disease and mortality, J Acquir Immune Defic Syndr 2014 November 28; 28(18): 2711-2719. doi:10.1097/QAD.0000000000000507. 
43. WHO: Consolidated guidelines on the use of antiretroviral drugs for treating and preventing HIV infection, Geneva, 2018, chapter 4 clinical guideline: Antiretroviral therapy, available at (http://www.who.int/hiv/pub/guidelines/arv2018/download/en).

44. Roja RY, Benu P, Rao SHB, Ramachandra MV, Simanchal P, Prasanna K. Predictors of Mortality among HIV Patients on HAART in an ART Centre - A Retrospective Study. Int J Med. Public Health. 2016; 6(4):175 - 9.

45. Ojikutu et al. Predictors of mortality in patients initiating antiretroviral therapy in Durban, South Africa, S Afr Med J. 2008 March ; 98(3): 204-208.

46. Amitabh B Suthar et al. Effect of cotrimoxazole on mortality in HIV-infected adults on antiretroviral therapy: a systematic review and meta-analysis, Bull World Health Organ 2012; 90:128-138C; doi:10.2471/BLT.11.093260.

47. Zheng et al. (2014) Incidence and risk factors for AIDS-related mortality in HIV patients in China: a cross-sectional study. BMC Public Health 2014 14:831. doi:10.1186/1471-2458-14-831.

\section{Tables}

[Please see the supplementary files section to view the tables.]

\section{Figures}

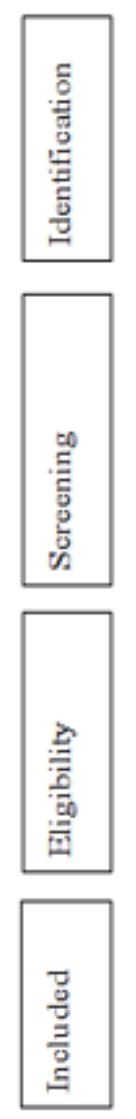

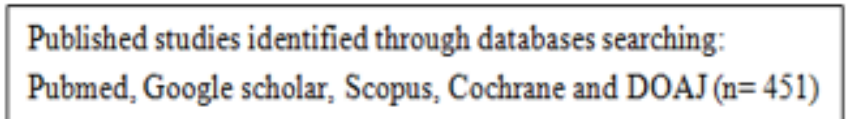
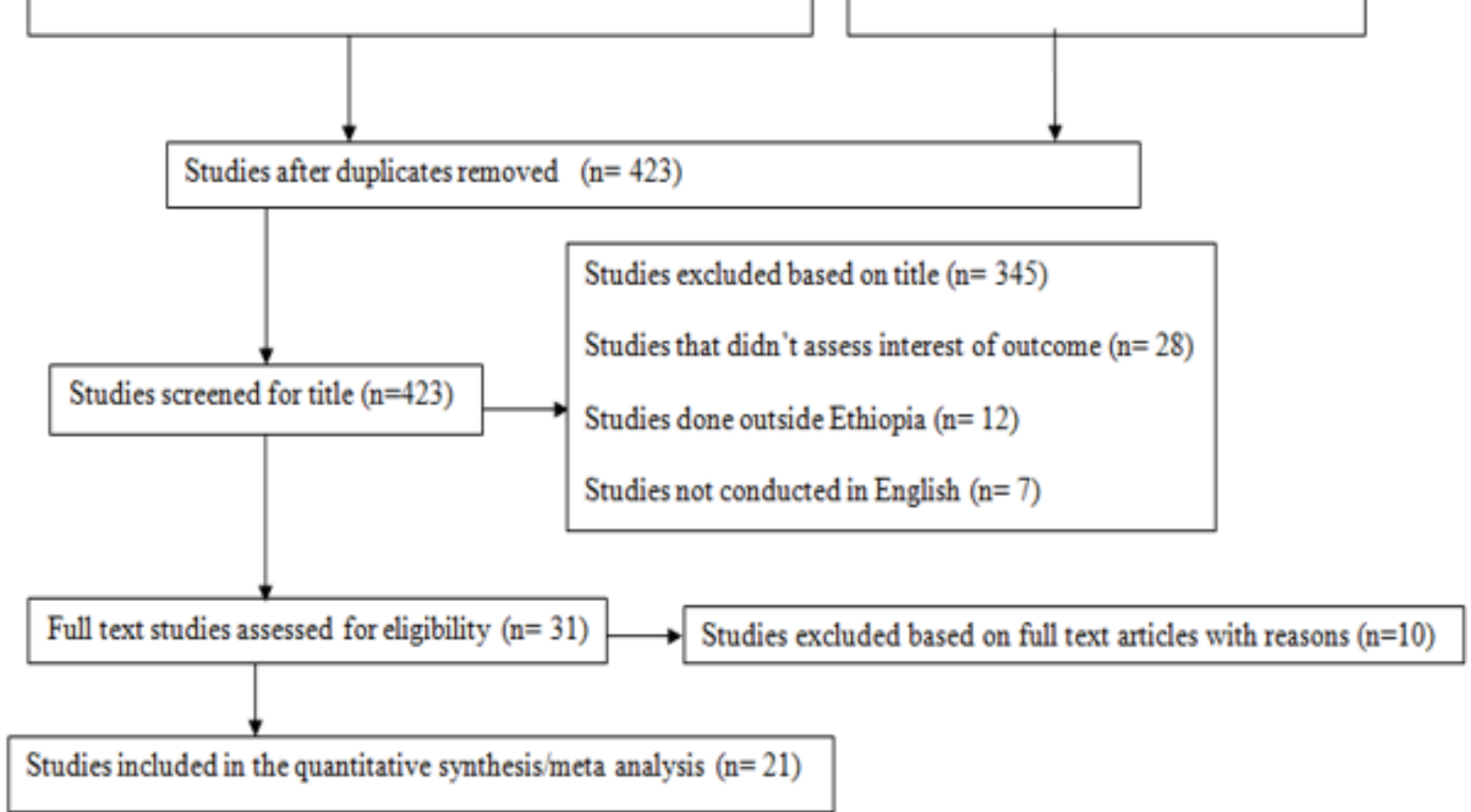
Figure 1

PRISMA flow diagram showing the procedures of studies selection on incidence and determinants of mortality on adult patients taking HAART in Ethiopia, 2019.

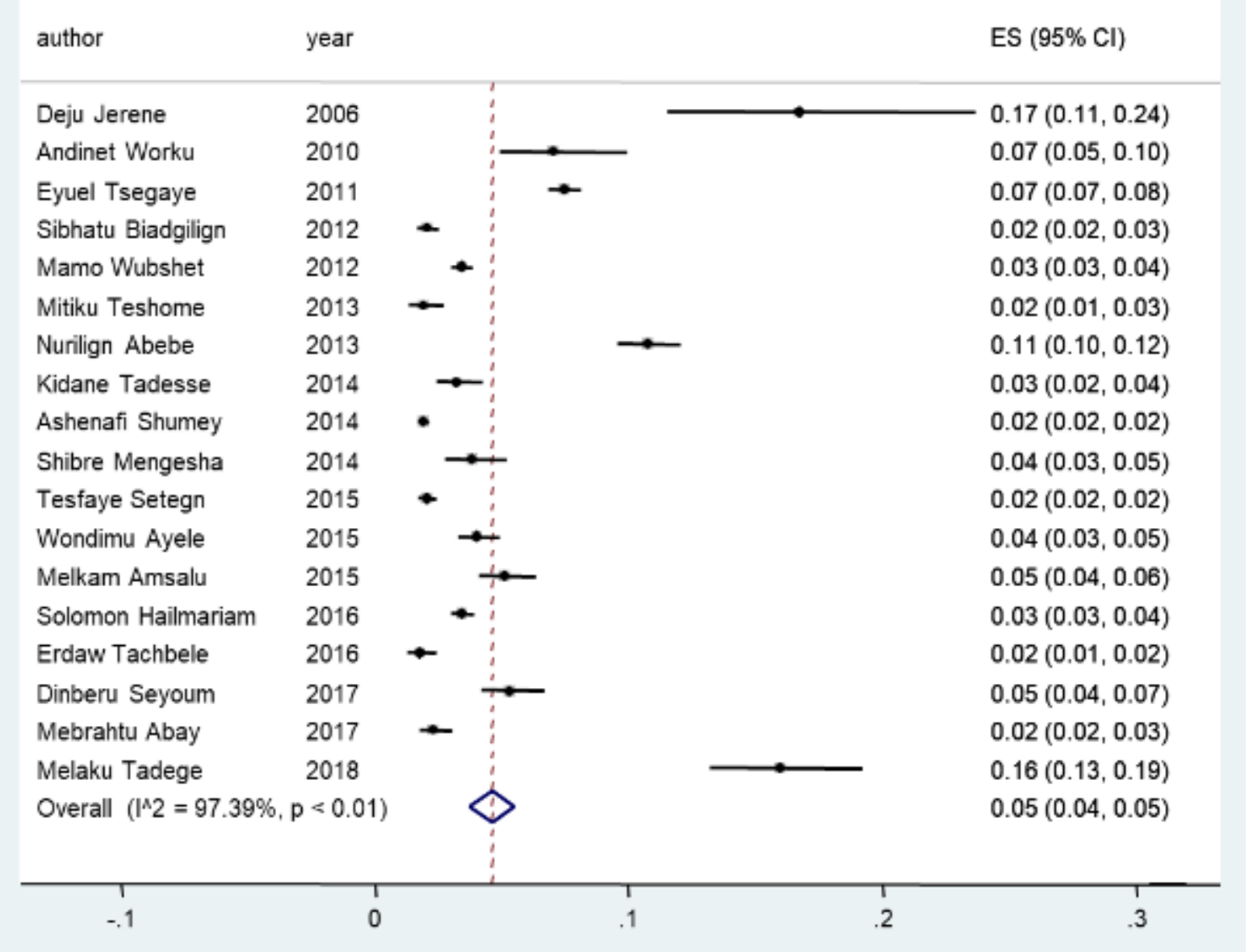

Figure 2

A forest plot showing the overall pooled estimate of incidence of mortality among studies done on adult HIV infected patients after HAART initiation in Ethiopia, 2019. 


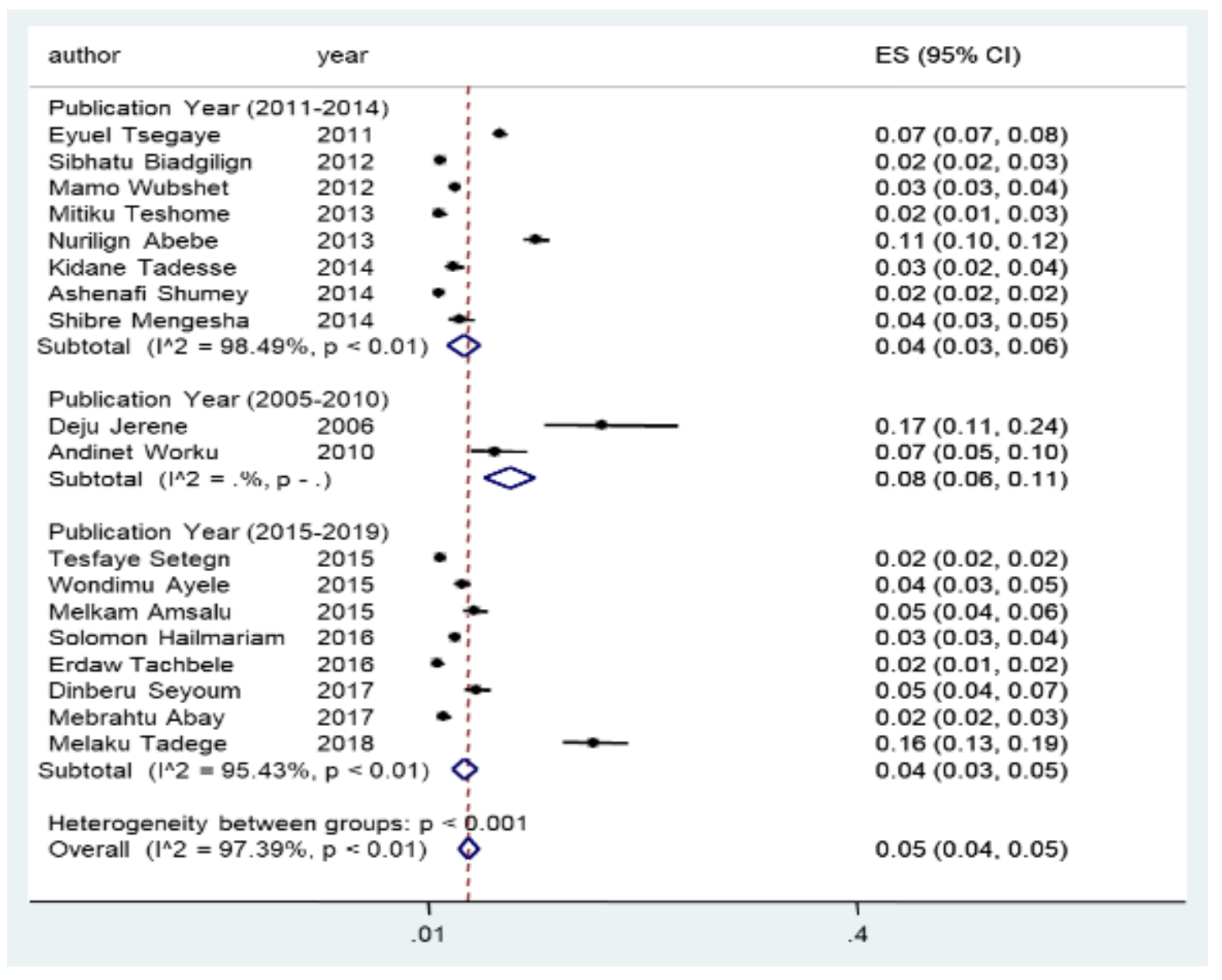

\section{Figure 3}

subgroup analysis of the incidence of mortality among HIV adult patients in Ethiopia, 2019

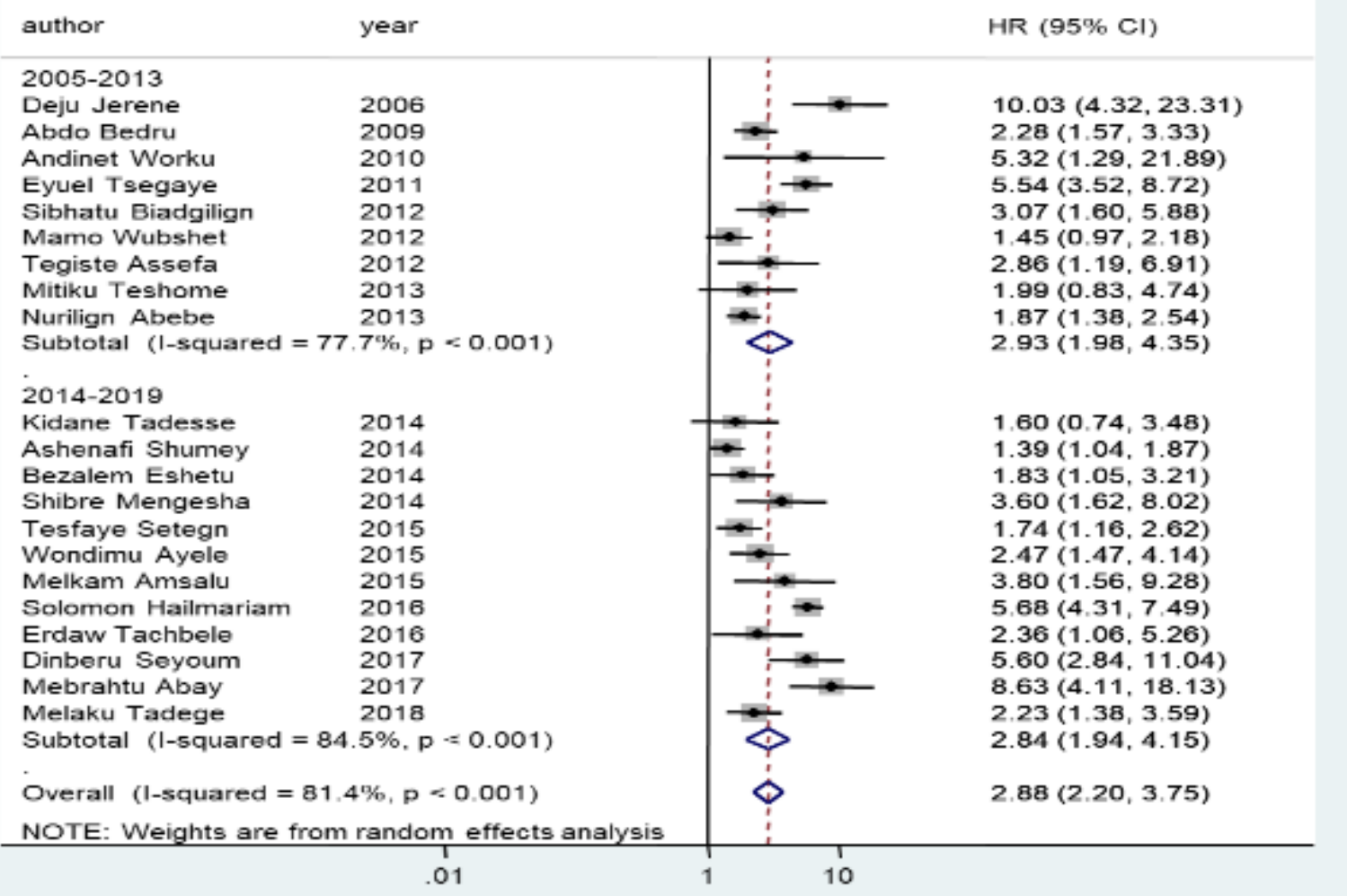


Figure 4

A forest plot of estimated hazard ratio of WHO clinical stage on adult patients after HAART initiation in Ethiopia, 2005-2019.

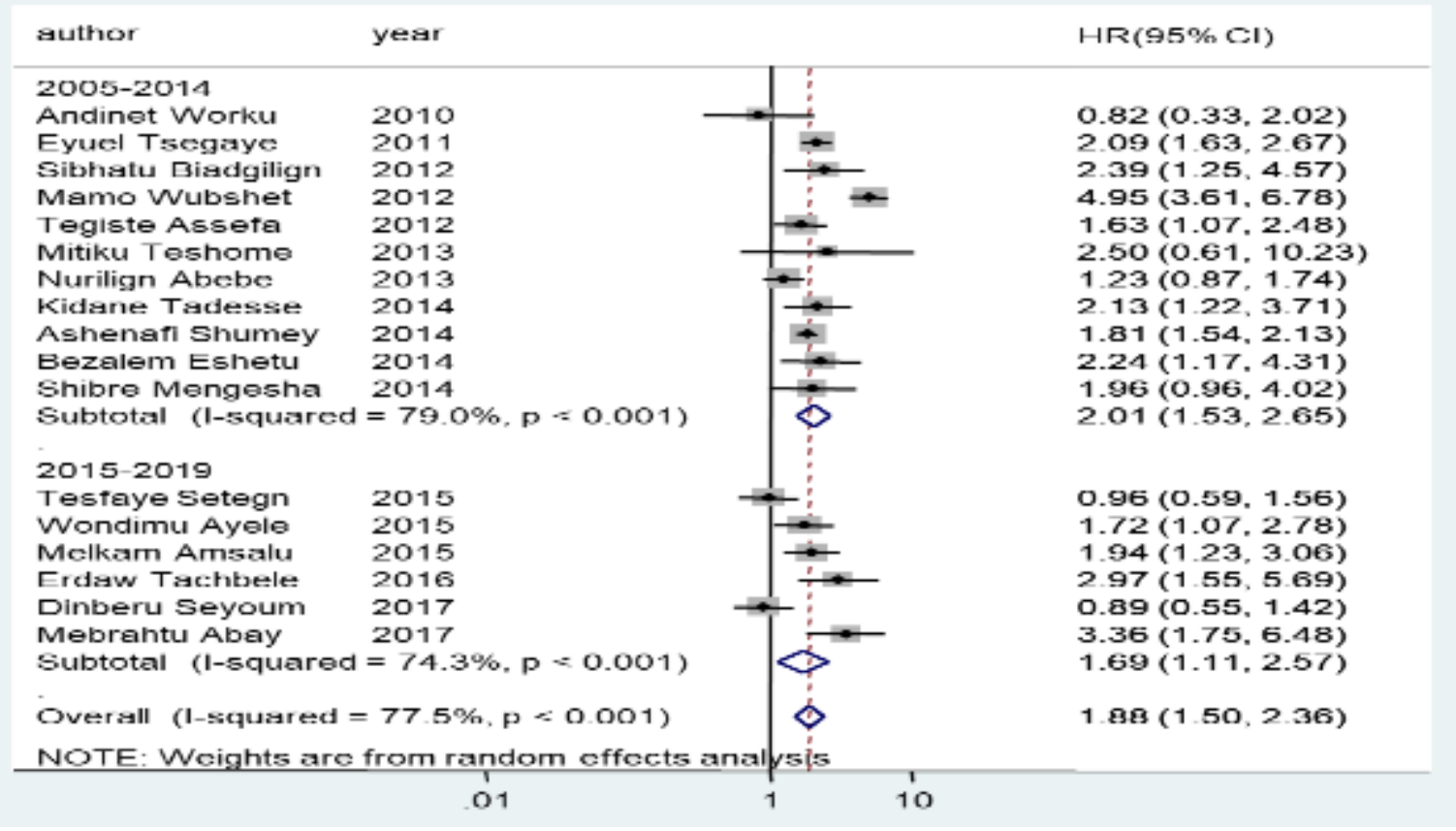

\section{Figure 5}

A forest plot showing the pooled hazard ratio of CD 4 counts on adult HIV infected patients after HAART initiation in Ethiopia, 2005-2019.

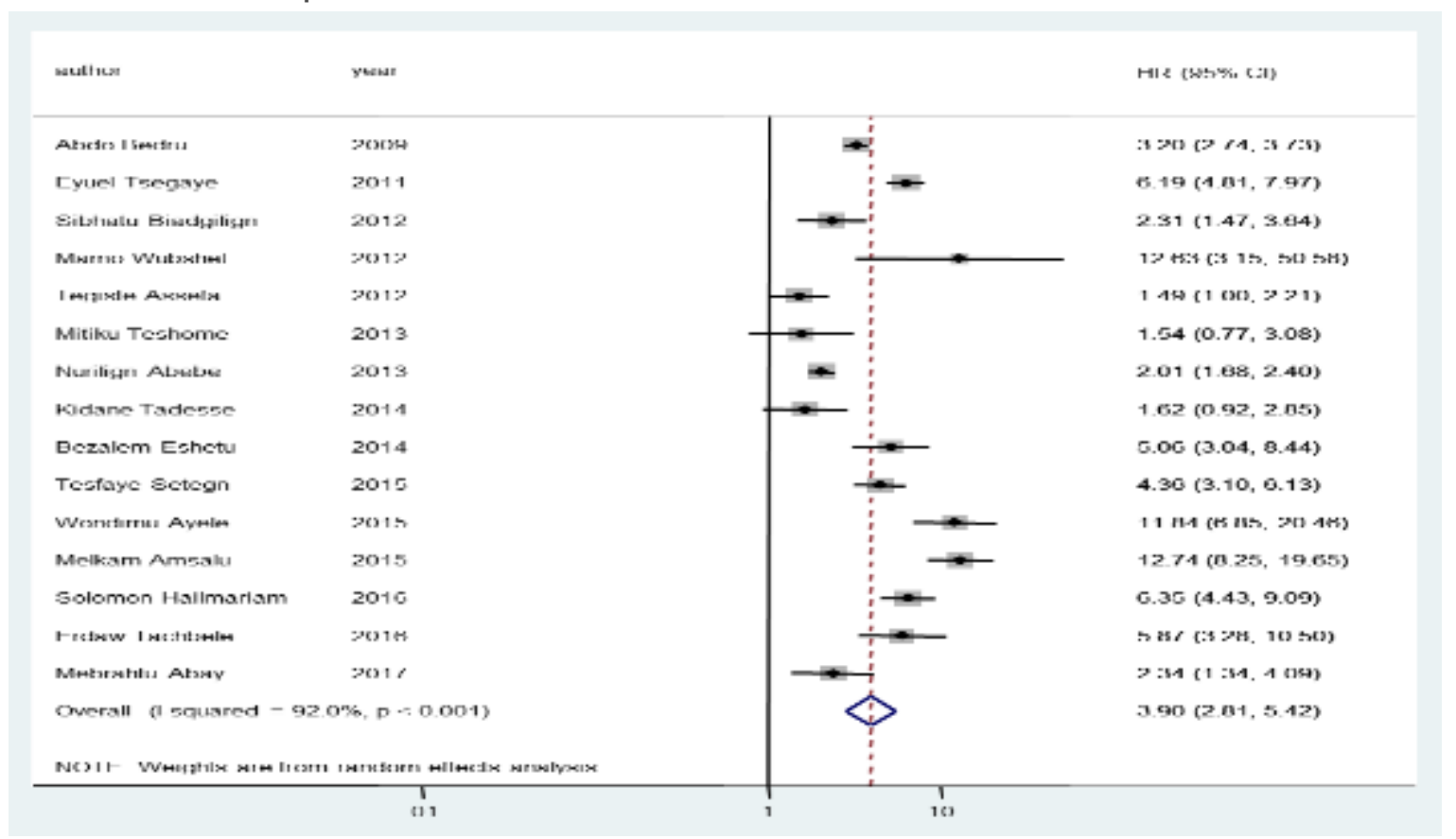

\section{Figure 6}


A forest plot of hazard ratio based on functional status on adult patients after HAART initiation in Ethiopia, 2019.

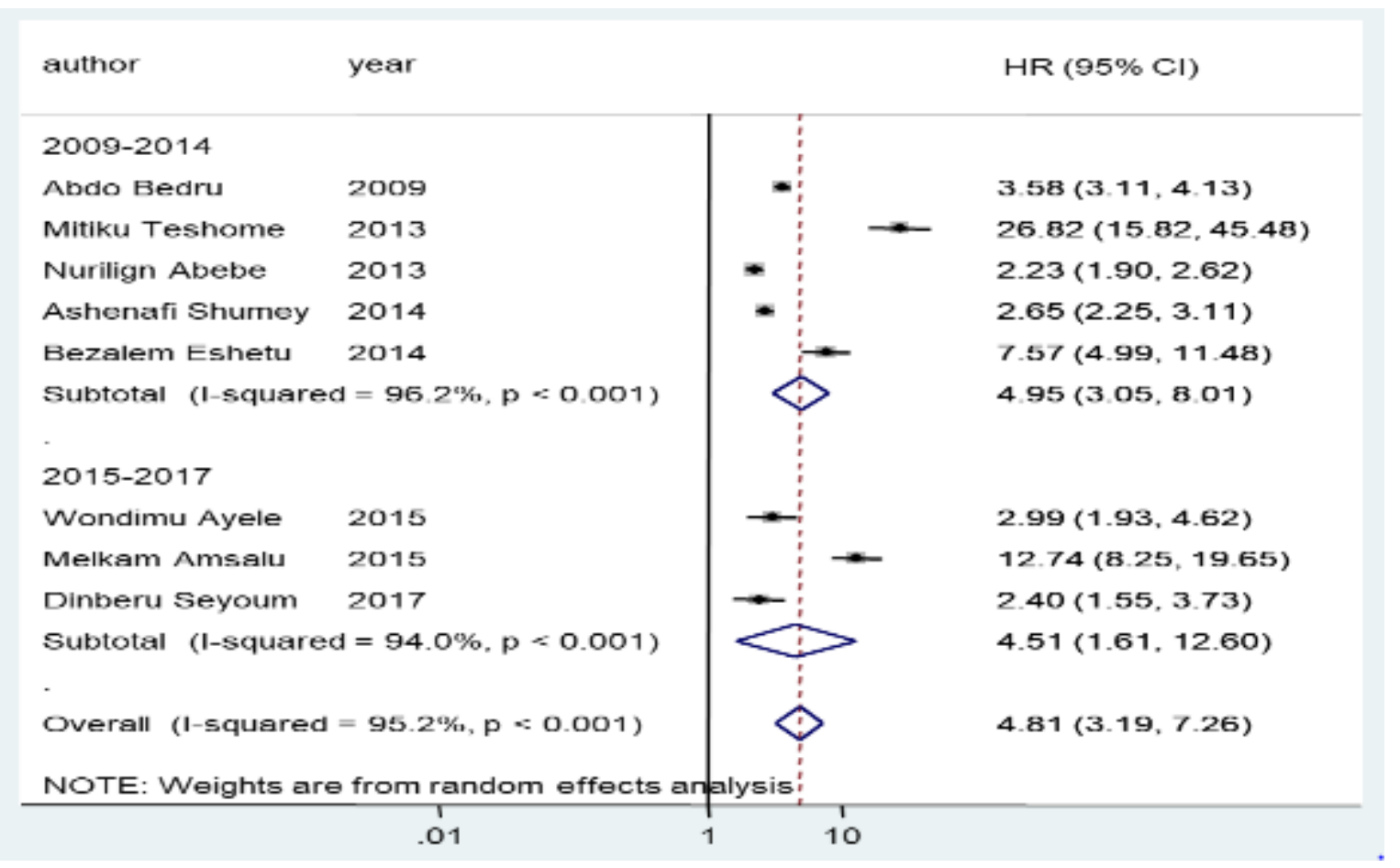

Figure 7

A forest plot estimate of hazard ratio drugs adherence in HIV mortality among adult patients in Ethiopia, 2019.

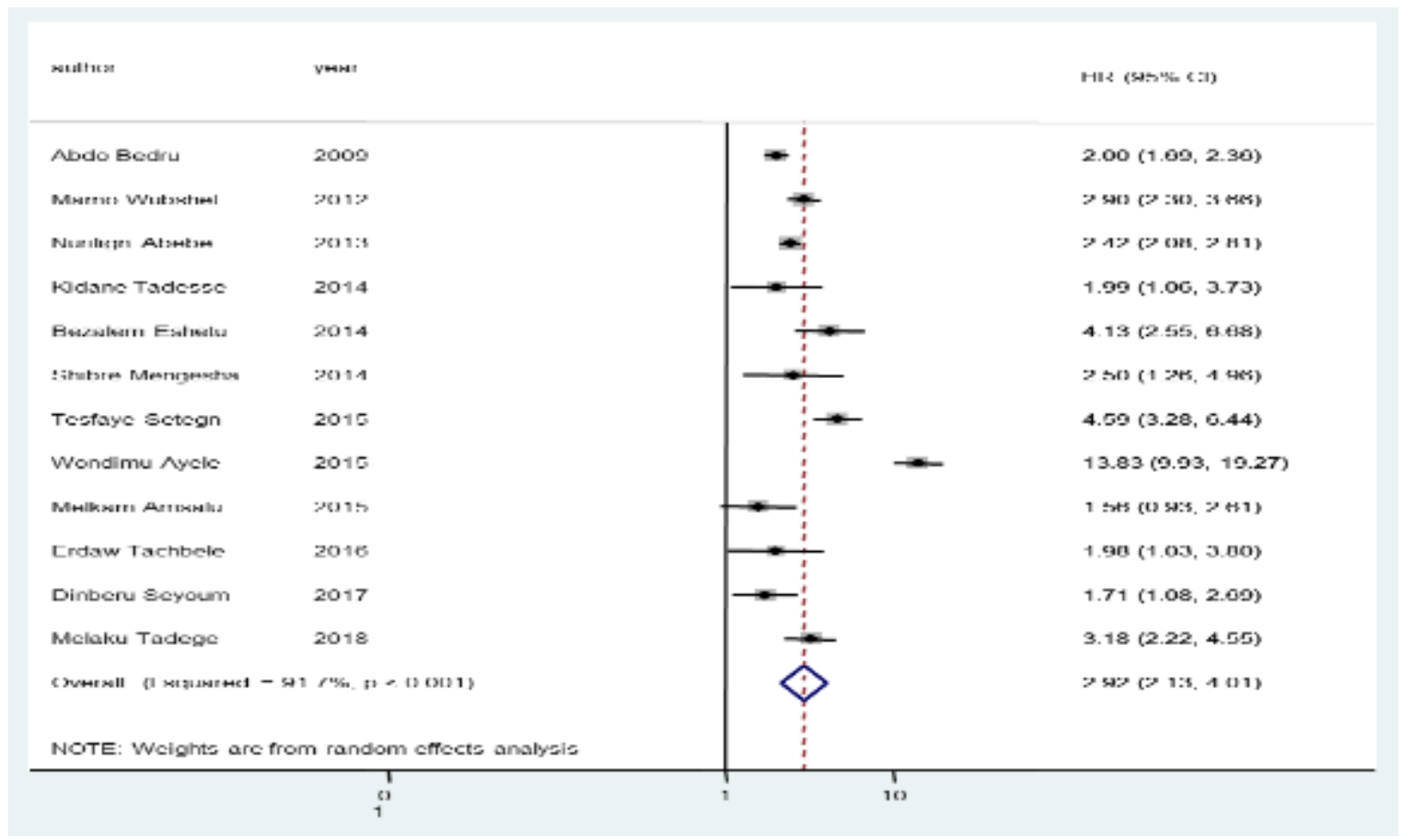

\section{Figure 8}


A forest plot showing the pooled estimate of hazard ratio of patients with tuberculosis after HAART initiation in Ethiopia, 2019.

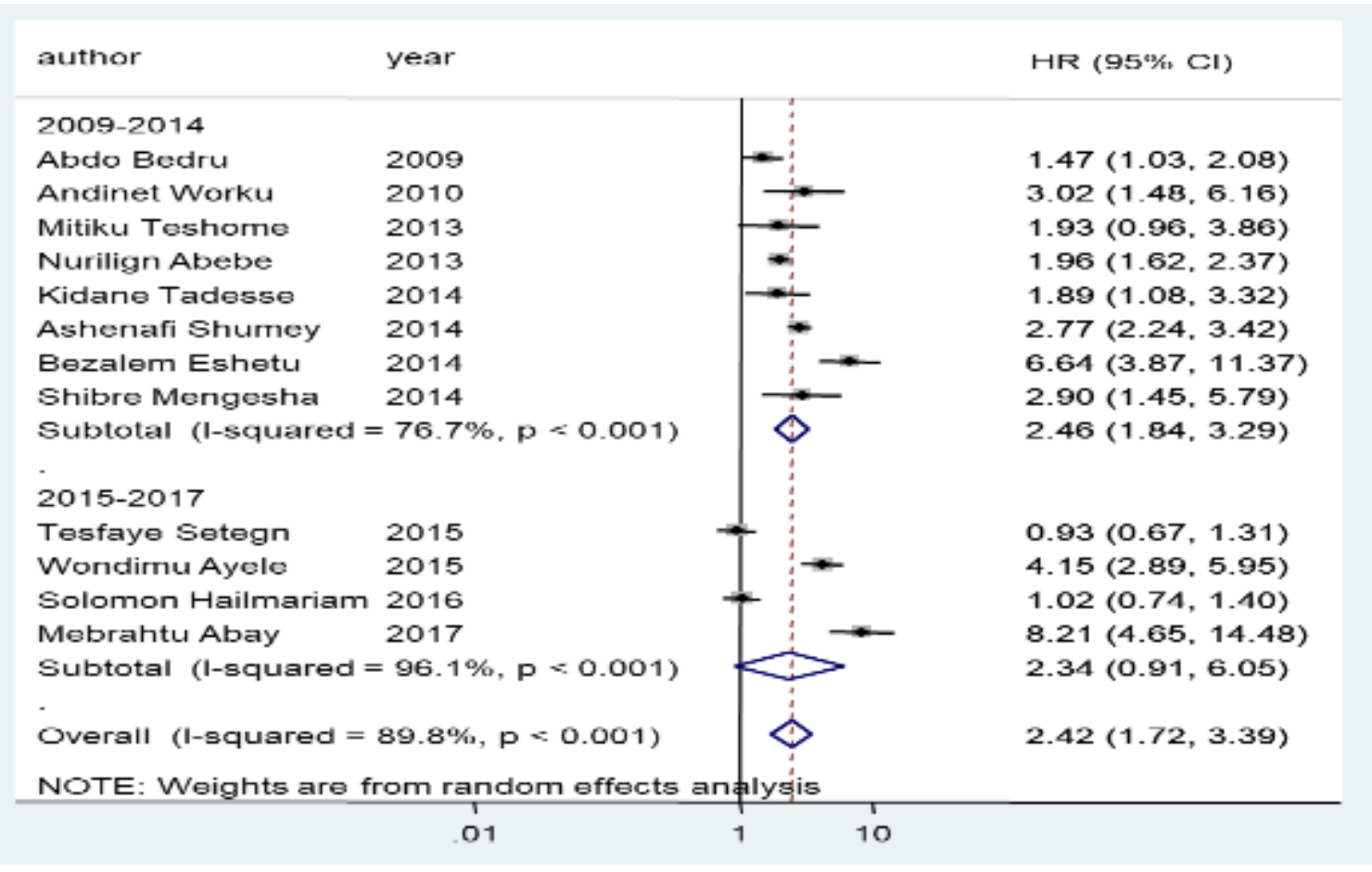

\section{Figure 9}

A forest plot showing hazard ratio of patients with hemoglobin level at initiation of HAART in Ethiopia, 2019.

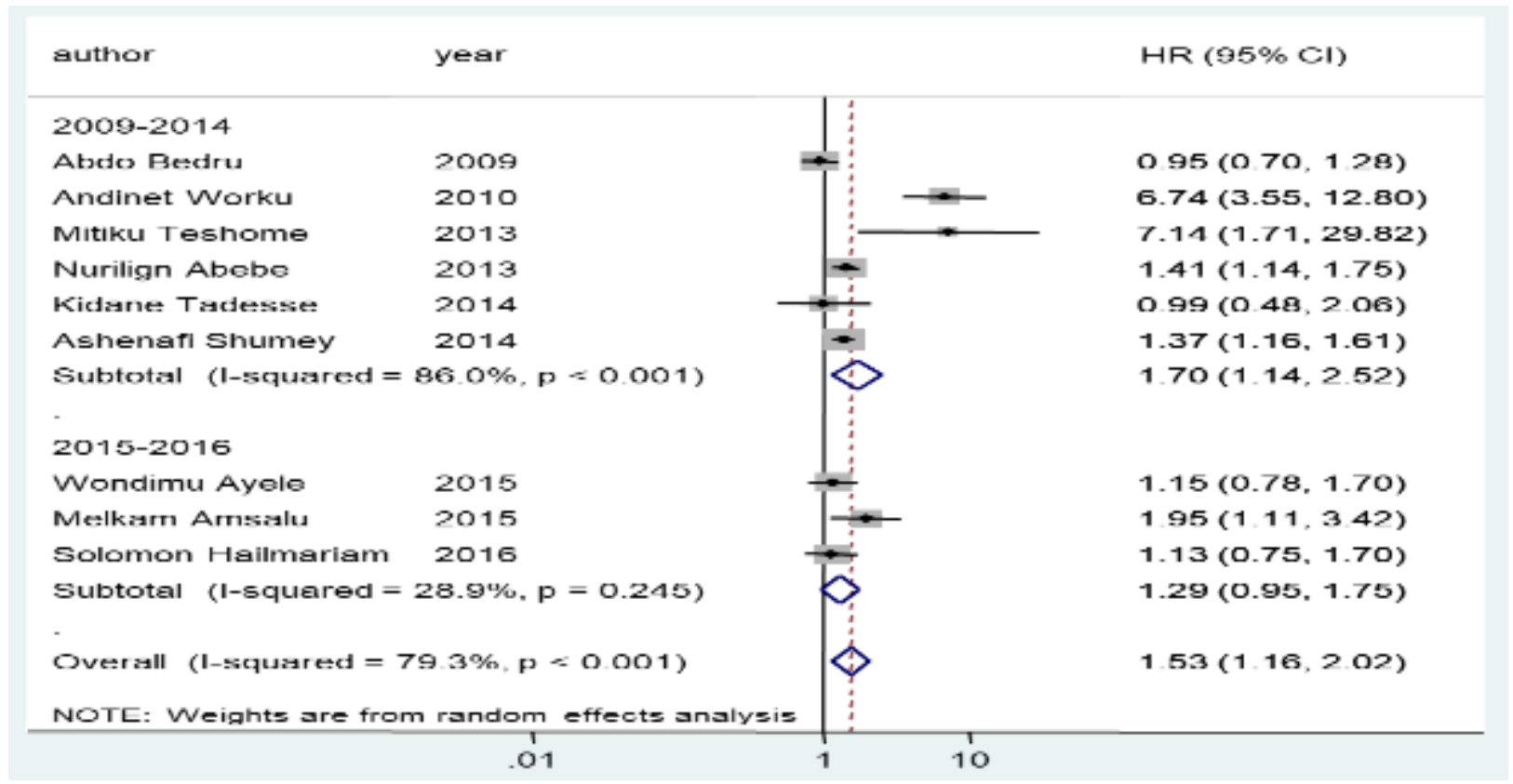

Figure 10

A forest plot hazard ratio of CPT on patients after HAART initiation in Ethiopia, 2019 


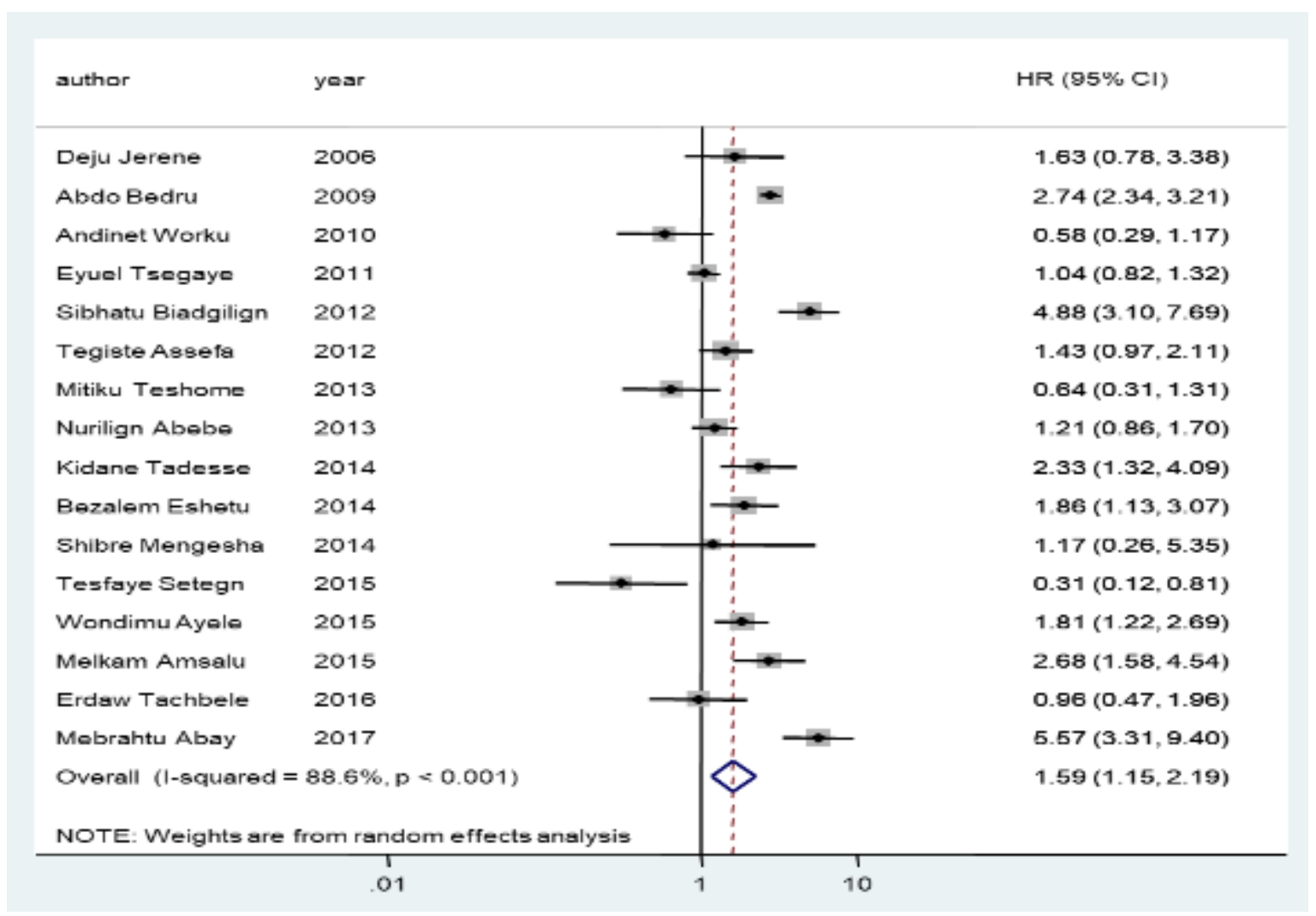

\section{Figure 11}

A forest plot of estimate of hazard ratio of body weight on patients after HAART initiation in Ethiopia, 2019.

\begin{tabular}{|c|c|c|}
\hline author & year & $\mathrm{HR}(95 \% \mathrm{Cl})$ \\
\hline Abda Bedru & 2009 & $1.12(0.95,1.33)$ \\
\hline Andinet Worku & 2010 & $1.53(0.76,3.09)$ \\
\hline Eyuel Tsegaye & 2011 & $1.63(1.37,1.93)$ \\
\hline Sibhatu Biadgilign & 2012 & $0.85(0.65,1.32)$ \\
\hline Mamo Wubshet & 2012 & $3.99(2.94,5.41)$ \\
\hline Tegiste $A=s e f a$ & 2012 & $1.42(0.98,2.07)$ \\
\hline Mitiku Teshome & 2013 & $1.59(0.80,3.17)$ \\
\hline Nurilign Abebe & 2013 & $1.03(0.85,1.24)$ \\
\hline Kidane Tadesse & 2014 & $1.43(0.82,2.48)$ \\
\hline Ashenafi Shumey & 2014 & $1.17(1.00,1.37)$ \\
\hline Bezalem Eshetu & 2014 & $1.81(1.11,2.97)$ \\
\hline Tesfaye Setegn & 2015 & $2.70(1.86,3.93)$ \\
\hline Wondimu Ayele & 2015 & $1.20(0.82,1.76)$ \\
\hline Melkam Amsalu & 2015 & $0.99(0.64,1.54)$ \\
\hline Solomon Hailmariam & 2016 & $1.16(0.89 .1 .52)$ \\
\hline Erdaw Tachbele & 2016 & $0.97(0.51,1.83)$ \\
\hline Dinberu Seyoum & 2017 & $1.32(0.84,2.09)$ \\
\hline Mebrahtu Abay & 2017 & $1.72(0.99 .2 .96)$ \\
\hline Melaku Tadege & 2018 & $1.28(0.87,1.86)$ \\
\hline \multicolumn{2}{|c|}{ Overall (1-squared $=81.1 \%, p=0.001)$} & $1.41(1.19,1.67)$ \\
\hline \multicolumn{2}{|c|}{ NOTE: Woights are from random offects analysis } & \\
\hline & .01 & \\
\hline
\end{tabular}


A forest plot of hazard ratio of gender on adult patients taking HAART in Ethiopia, 2019.

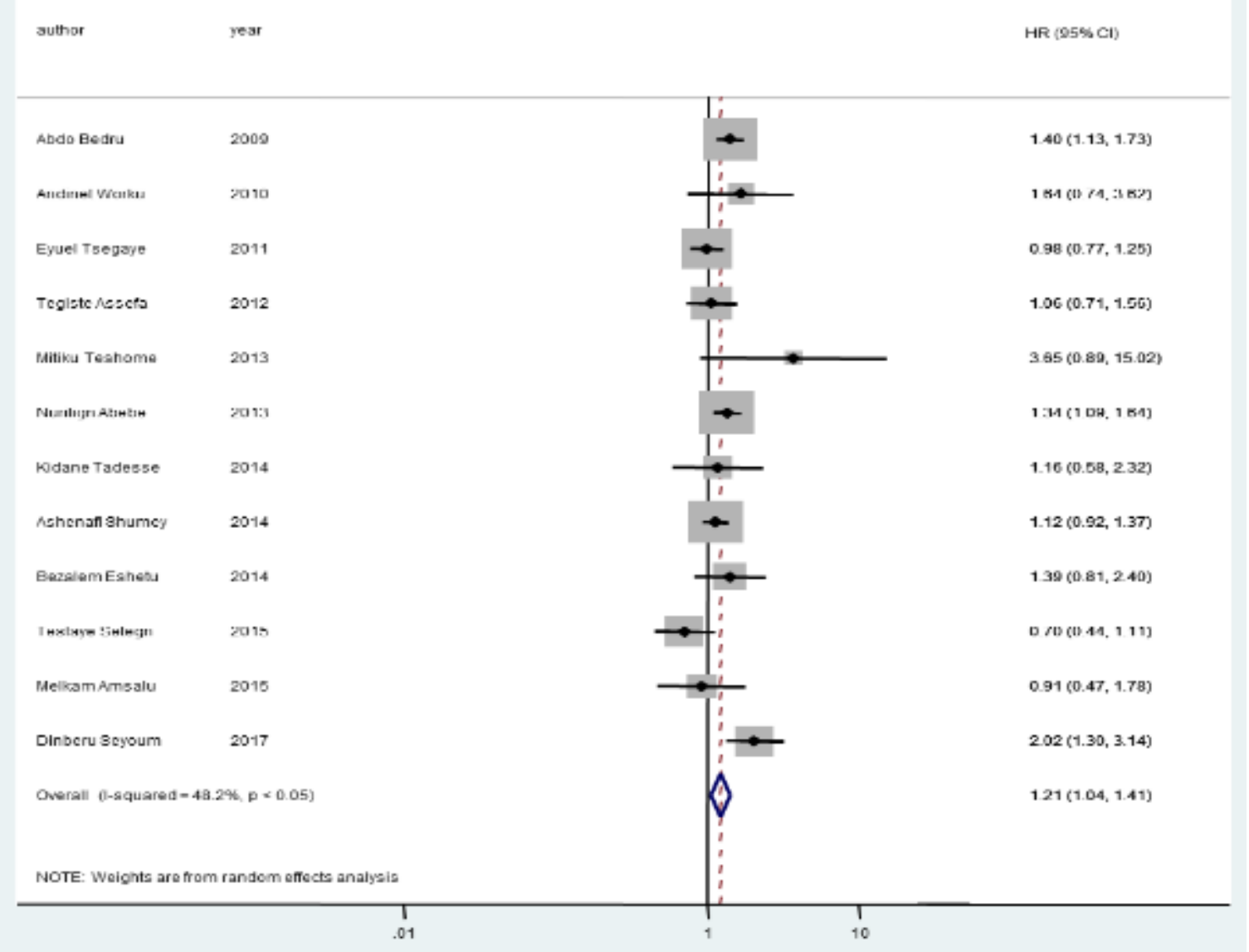

Figure 13

A forest plot of hazard ratio of age on adult patients after taking HAART in Ethiopia, 2019.

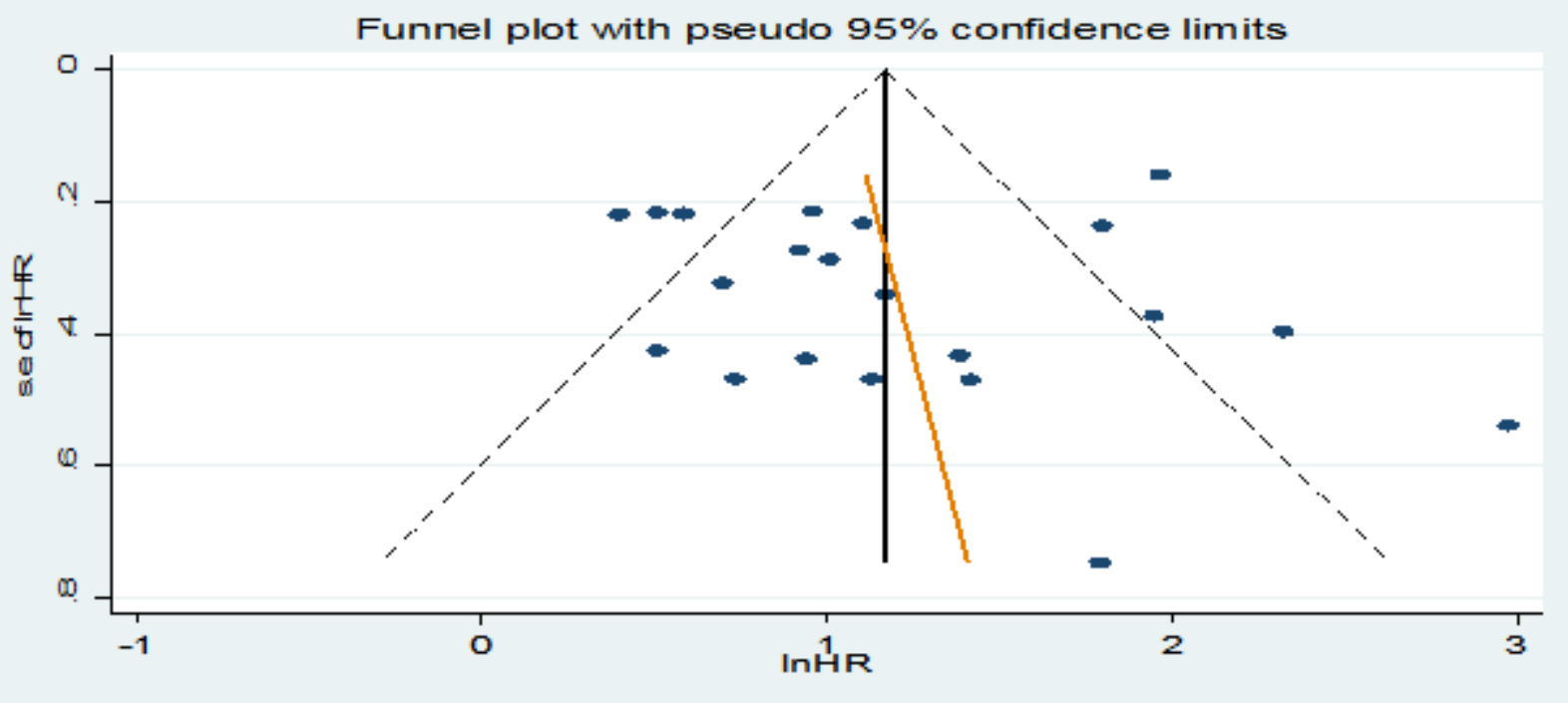

Figure 14 
A funnel plot of log of a hazard ratio with its standard error in log form assessing publication bias in the included studies in Ethiopia, 2019.

\section{Supplementary Files}

This is a list of supplementary files associated with this preprint. Click to download.

- Tables.docx

- PRISMA2009checklistforadditionaldocument.doc 